\section{Incidência de lesões laríngeas não neoplásicas em pacientes com queixas vocais}

\author{
Erich Christiano Madruga de Melo', \\ Lupercio Luz Brito', \\ Osiris Camponês Oliveira Brasil', Mara Beblau, \\ Danielly Madruga de Melo ${ }^{4}$
}

\section{Resumo}

I

ntrodução: As lesões benignas que acometem o trato vocal, em especial as pregas vocais, são comuns e produzem em sua maioria uma sintomatologia caracterizada por disfonia. Observa-se que mais de $50 \%$ das pessoas com queixa vocal apresentam alteração benigna da mucosa das pregas vocais. Existem poucos trabalhos na literatura sobre a real incidência das diferentes lesões benignas laríngeas, sendo, em sua maioria, relatos de séries representativas de achados cirúrgicos de microcirurgia da laringe. Objetivo: Este estudo foi realizado com o objetivo de avaliar a incidência das lesões laríngeas não-neoplásicas em pacientes com queixas vocais avaliados no Hospital do Servidor Público do Estado de São Paulo - Francisco Morato de Oliveira, no período de março de 1999 a março de 2000. Forma de estudo: Retrospectivo não randomizado. Material e método: Foram feitas análises da incidência por sexo e idade, avaliando-se também o tipo da lesão, sua ocorrência de forma isolada ou associada, tipo de coaptação glótica e presença de sinais sugestivos de refluxo gastresofágico. Foram reavaliados 1093 exames de videolaringoscopia, sem estroboscopia, de pacientes com queixas vocais. A amostra foi composta de 131 exames de pacientes do sexo masculino e 962 do sexo feminino, com idade variando de 4 a 80 anos, com média de 42,5 anos. Resultados: Foram identificadas 13 alterações laríngeas, a saber: cisto, 24\%; edema de Reinke, $10 \%$; sulco vocal, 10\%; nódulo vocal, 8\%; lesão nodular, 8\%; pólipo, 7\%; vasculodisgenesia, 4\%; paralisia de pregas vocais, 3\%; laringite, $3 \%$; leucoplasia, $2 \%$; granuloma, $2 \%$; micromembrana anterior, $1 \%$; ponte de mucosa, $<1 \%$. O exame foi avaliado como normal em 18\% dos indivíduos. Os autores também descrevem a distribuição dos 1093 exames quanto à idade e ao sexo dos pacientes e às demais variáveis analisadas.

\section{Incidence of non-neoplasic lesion in patients with vocal complains}

Palavras-chave: disfonia, incidência, laringe. Key words: dysphonia, incidence, larynx.

\section{Summary}

\begin{abstract}
ntroduction: Benign lesions of the larynx are commonly seen and can frequently produce dysphonia as a characteristic signal. More than $50 \%$ of patients with a benign laryngeal alteration presents a vocal complain. There are few available studies on the real incidence of these lesions; being usually reports on microsurgical findings during laryngeal direct intervention. Aim: The goal of the present study was to evaluate the incidence of non-neoplasic benign lesions, in patients with vocal complains, seen at the "Hospital do Servidor Público do Estado de São Paulo - Francisco Morato de Oliveira", during a one year period, from March 1999 to March 2000. Study design: Retrospective no randomized. Material and method: The parameters considered for the analysis were sex, age, type of lesion (isolated or in association with other findings), type of glottic closure and presence of suggestive signs of GER. 1093 videolaryngoscopic examinations, from patients with vocal complains, without stroboscopy, were considered for the analysis, being 131 examinations from male patients and 962 from female patients, age distribution from 4 to 80 years-old, mean age of 42.5 years. Results: 13 different laryngeal pathologies were identified: vocal fold cyst 24\%; Reinke's edema 10\%; vocal fold sulcus $10 \%$; vocal fold nodules $8 \%$; nodular lesion $8 \%$; polyps $7 \%$; vasculodysgenesy $4 \%$; vocal fold paralysis 3\%; laryngitis $3 \%$; leukoplaquia $2 \%$; granulomma $2 \%$; anterior microweb $1 \%$; mucosal bridge $<1 \%$. Normal evaluation was achieved by $18 \%$ of patients. This study also describes the distribution of the 1093 cases according to age, sex and other studied parameters.
\end{abstract}

\footnotetext{
${ }^{1}$ Medico Residente do Serviço de Otorrinolaringologia do Hospital do Servidor Público Estadual de São Paulo / FMO

${ }^{2}$ Medico Chefe do Setor de Cirurgia de Cabeça e Pescoço do Serviço de Otorrinolaringologia do Hospital do Servidor Público Estadual de São Paulo / FMO; Doutor em Medicina pela Escola Paulista de Medicina - Unifesp

${ }^{3}$ Fonoaudióloga; Doutora em Ciências pela Universidade Federal de São Paulo - EPM; Diretora do Centro de Estudos da Voz - CEV, São Paulo ${ }^{4}$ Graduanda do Curso de Fonoaudiologia do Centro Paraibano de Educação

Hospital do Servidor Público Estadual de São Paulo / Francisco Morato de Oliveira - São Paulo - SP

Erich Christiano Madruga de Melo - R. Borges Lagoa, 1565 Apto. 10 - Vila Clementino - São Paulo - SP - 04038-034 - E-mail: erichmelo@uol.com.br Artigo recebido em 03 de abril de 2001. Artigo aceito em 27 de abril de 2001.
} 


\section{INTRODUÇÃO}

As desordens vocais são caracterizadas por desvios que comprometem a inteligibilidade e a efetividade da comunicação oral, dentre os quais podemos incluir alterações na qualidade vocal, freqüência e intensidade da voz, por desordens no funcionamento laríngeo, respiratório e/ou do trato vocal ${ }^{14}$.

As lesões benignas que podem acometer o trato vocal, em especial as pregas vocais, são comuns e produzem em sua maioria uma sintomatologia caracterizada por disfonia ${ }^{6}$. Mais de $50 \%$ das pessoas com queixa vocal apresentam alteração benigna da mucosa das pregas vocais $^{1}$.

Brodnitz (1963) relatou em 977 pacientes com queixa vocal uma incidência de $45 \%$ de nódulo, pólipo e edema de Reinke. Kleinsasser (1979) relatou que mais de 50\% de 2618 pacientes tinham uma destas entidades diagnósticas.

Ramig \& Verdolini (1998) relataram a ocorrência das desordens vocais na população infantil, adulta e da terceira idade como sendo respectivamente: de 3-6\% a 23,4\%; 3 a 9\%; e 12 a 35\%. As autoras ainda ressaltam que, nos indivíduos que fazem uso crítico da voz no trabalho, a incidência de alterações vocais pode chegar a 25\%. A importância da voz é tamanha, que 3,29\% dos indivíduos trabalhadores dependem dela para promover a segurança pública.

Tabela 1. Distribuição dos 1093 exames quanto a idade, sexo e tipo de lesão encontrada.

\begin{tabular}{lcc}
\hline Idade & $\begin{array}{c}04 \text { a } 80 \\
\text { anos }\end{array}$ & $\begin{array}{c}\text { média }=42,56 \\
\text { anos }\end{array}$ \\
\hline Sexo & $\mathrm{N}$ & $\%$ \\
\hline Masculino & 131 & 12 \\
Feminino & 962 & 88 \\
\hline Tipo de Lesão & $\mathrm{N}$ & $\%$ \\
\hline I - $\quad$ AEM & 430 & 39 \\
$\quad$ Cisto & $267(267 / 430=62 \%)$ & 24 \\
$\quad$ Sulco (Estrias e Bolsa) & $110(110 / 430=26 \%)$ & 10 \\
$\quad$ Ponte De Mucosa & $1(1 / 430=0 \%)$ & 0 \\
$\quad$ Micromembrana & $10(10 / 430=2 \%)$ & 1 \\
$\quad$ Vasculodisgenesia & $42(42 / 430=10 \%)$ & 4 \\
II - $\quad$ Nódulo & 90 & 8 \\
III - Lesão Nodular & 88 & 8 \\
IV - Pólipos & 75 & 7 \\
V - Edema de Reinke & 113 & 10 \\
VI - Leucoplasias & 17 & 2 \\
VII - Granulomas & 18 & 2 \\
VIII - Paralisias & 33 & 3 \\
IX - Laringites & 33 & 3 \\
X - Exame Normal & 196 & 18 \\
\hline
\end{tabular}

Os indivíduos disfônicos consideram que os problemas vocais afetam negativamente o seu funcionamento social e sua estabilidade emocional. A voz reflete características da personalidade do falante e, por este motivo, uma desordem vocal pode ter efeitos devastadores em indivíduos de todas as idades ${ }^{14}$.

Existem poucos trabalhos na literatura sobre a real incidência das diferentes lesões benignas laríngeas, sendo, em sua maioria, relatos de séries representativas de achados cirúrgicos de microcirurgia da laringe $e^{3,5,12}$

Este estudo foi realizado com o objetivo de avaliar a incidência das lesões laríngeas não-neoplásicas, em pacientes com queixas vocais avaliados no Hospital do Servidor Público do Estado de São Paulo - Francisco Morato de Oliveira, no período de um ano, de março de 1999 a março de 2000. Foram feitas análises da incidência por sexo e idade, avaliando-se também o tipo da lesão, sua ocorrência de forma isolada ou associada, tipo de coaptação glótica e presença de sinais sugestivos de refluxo gastresofágico.

\section{MATERIAL E MÉTODO}

\section{Material}

Foram reavaliados todos os exames de videolaringoscopia, sem estroboscopia, de pacientes com queixas vocais, realizados no Hospital do Servidor Público do Estado de São Paulo, no período de março de 1999 a

Tabela 2. Achados nos pacientes portadores de cisto vocal.

\begin{tabular}{lcc}
\hline Idade & $\begin{array}{c}10 \text { a } 80 \\
\text { anos }\end{array}$ & $\begin{array}{c}\text { Média }=39,99 \\
\text { anos }\end{array}$ \\
\hline Sexo & $\mathrm{N}$ & $\%$ \\
\hline Masc & 9 & 3 \\
Fem & 258 & 97 \\
\hline & $\mathrm{N}$ & $\%$ \\
\hline Lesão Isolada & 223 & 84 \\
Lesão Associada & 44 & 16 \\
Vasculodisgenesia & 35 & 80 \\
Micromembrana Laríngea & 5 & 11 \\
Sulco & 3 & 7 \\
Leucoplasia & 1 & 2 \\
Fenda Glótica & $\mathrm{N}$ & 1 \\
Triangular Posterior & 2 & 3 \\
Triang.médio-posterior & 7 & 6 \\
Dupla & 16 & 29 \\
Ampulheta & 76 & 5 \\
Fusiforme & 14 & 12 \\
Irregular & 32 & 4 \\
Paralela & 10 & 23 \\
Sinais de Refluxo Gastresofágico & 61 & \\
\hline
\end{tabular}

AEM: Alteração Estrutural Mínima 
março de 2000. A amostra foi composta por 1093 exames, sendo 131 de pacientes do sexo masculino e 962 do sexo feminino, com idade variando de 4 a 80 anos, com média de 42,5 anos.

\section{Método}

Os exames foram realizados por médicos do Setor de Laringologia do Serviço de Otorrinolaringologia. Os diagnósticos clínicos foram baseados na impressão visual das lesões, sendo o nódulo vocal caracterizado por uma lesão de massa exofítica, bilateral, simétrica em localização mas não necessariamente em tamanho, mais ou menos flexível, com certa mobilidade durante a emissão vocal; já o cisto vocal é uma lesão mais intra-cordal, uni ou bilateral, podendo ser assimétrica em localização e tamanho, mais rígida e com menor mobilidade durante a emissão vocal.

Foram analisados o tipo de lesão, se isolada ou associada, o tipo de coaptação glótica e a presença de sinais sugestivos de refluxo gastresofágico. Nesta última avaliação foram considerados como sinais sugestivos: edema retrocricóide, espessamento de mucosa interaritenóidea, hiperemia de região de cartilagens aritenóideas, úlcera e/ou granuloma de região posterior.

$\mathrm{Na}$ videolaringoscopia foram utilizados os seguintes equipamentos:

- telescópio de laringe rígido de 7,0 $\mathrm{mm}$ de $70^{\circ}$ (RICHARDS WOLF);

Tabela 3. Achados nos pacientes portadores de Edema de Reinke.

\begin{tabular}{|c|c|c|}
\hline Idade & $\begin{array}{c}26 \text { a } 76 \\
\text { anos }\end{array}$ & $\begin{array}{c}\text { Média }=51,08 \\
\text { anos }\end{array}$ \\
\hline Sexo & $\mathrm{N}$ & $\%$ \\
\hline Masc & 5 & 4 \\
\hline \multirow[t]{2}{*}{ Fem } & 108 & 96 \\
\hline & $\mathrm{N}$ & $\%$ \\
\hline Lesão Isolada & 96 & 85 \\
\hline Lesão Associada & 17 & 15 \\
\hline Leucoplasia & 6 & 35 \\
\hline Vasculodisgenesia & 3 & 18 \\
\hline Laringite Crônica & 3 & 18 \\
\hline Pólipo & 2 & 12 \\
\hline Sulco & 2 & 12 \\
\hline Cisto & 1 & 5 \\
\hline Fenda Glótica & $\mathrm{N}$ & $\%$ \\
\hline Triangular Posterior & 2 & 2 \\
\hline Triang.médio-posterior & 1 & 1 \\
\hline Dupla & 0 & 0 \\
\hline Ampulheta & 2 & 2 \\
\hline Fusiforme & 1 & 1 \\
\hline Irregular & 29 & 25 \\
\hline Paralela & 1 & 1 \\
\hline Sinais de Refluxo Gastresofágico & 39 & 35 \\
\hline
\end{tabular}

- nasofibrolaringoscópio flexível de 3,2 mm (OLYMPUS ENF Type P3);

- fonte de luz Hi-light 250 watts (RICHARDS, SMITH +NEPHEW);

- micro-camêmra (RICHARDS WOLF, 5511 1CCD ENDOCAM);

- videocassete (SEMP, X682);

- monitor de vídeo (SONY HR, TRINITON PVM 145 3MD); - microfone (LENON ML8).

Os exames videolaringoscópicos foram realizados sob anestesia tópica com lidocaína 10\%, tendo-se utilizado o telescópio nos pacientes colaboradores e o nasofibroscópio nos demais; os exames foram registrados em fita VHS.

\section{RESULTADOS}

A distribuição dos 1093 exames quanto à idade e ao sexo dos pacientes e às demais variáveis analisadas encontra-se na Tabela 1. Foram identificadas 13 alterações laríngeas, a saber: cisto, 24\%; edema de Reinke, 10\%; sulco vocal, 10\%; nódulo vocal, 8\%; lesão nodular, 8\%; pólipo, 7\%; vasculodisgenesia, 4\%; paralisia de pregas vocais, 3\%; laringite, 3\%; leucoplasia, 2\%; granuloma, 2\%; micromembrana anterior, $1 \%$; ponte de mucosa, $<1 \%$. O exame foi considerado normal em 18\% das avaliações.

Os achados nos pacientes portadores das diferentes alterações encontram-se nas Tabelas de 2 a 11.

Tabela 4. Achados nos pacientes portadores de sulco vocal.

\begin{tabular}{lcc}
\hline Idade & $\begin{array}{c}24 \text { a } 72 \\
\text { anos }\end{array}$ & $\begin{array}{c}\text { Média }=42,87 \\
\text { anos }\end{array}$ \\
\hline Sexo & $\mathrm{N}$ & $\%$ \\
\hline Masc & 21 & 19 \\
Fem & 89 & 81 \\
\hline Tipo de Sulco & $\mathrm{N}$ & $\%$ \\
\hline Estria Menor & 70 & 64 \\
Estria Maior & 19 & 17 \\
Bolsa & 21 & 19 \\
Lesão Isolada & 78 & 71 \\
Lesão Associada & 32 & 29 \\
Cisto & 13 & 42 \\
Vasculodisgenesia & 13 & 42 \\
Pólipo & 3 & 8 \\
Granuloma & 3 & 8 \\
\hline Fenda Glótica & $\mathrm{N}$ & $\%$ \\
\hline Triangular Posterior & 0 & 0 \\
Triang.médio-posterior & 0 & 0 \\
Dupla & 2 & 24 \\
Ampulheta & 0 & 0 \\
Fusiforme & 26 & 19 \\
Irregular & 34 & 24 \\
Paralela & & 0 \\
\hline Sinais de Refluxo Gastresofágico & 20 & 0 \\
\hline
\end{tabular}


Tabela 5. Achados nos pacientes portadores de nódulo vocal.

\begin{tabular}{lcc}
\hline Idade & $\begin{array}{c}4 \text { a } 66 \\
\text { anos }\end{array}$ & $\begin{array}{c}\text { Média }=34,39 \\
\text { anos }\end{array}$ \\
\hline Sexo & $\mathrm{N}$ & $\%$ \\
\hline Masc & 12 & 13 \\
Fem & 78 & 87 \\
\hline \multicolumn{1}{c}{ Lesão Isolada } & $\mathrm{N}$ & $\%$ \\
Lesão Associada & 87 & 97 \\
$\quad$ Vasculodisgenesia & 3 & 3 \\
$\quad$ Micromembrana & 2 & 67 \\
\hline Fenda Glótica & 1 & 33 \\
\hline \multicolumn{1}{c}{ Triangular Posterior } & $\mathrm{N}$ & $\%$ \\
$\quad$ Triang.médio-posterior & 2 & 2 \\
$\quad$ Dupla & 25 & 28 \\
Ampulheta & 33 & 37 \\
$\quad$ Fusiforme & 9 & 10 \\
$\quad$ Irregular & 2 & 2 \\
Paralela & 3 & 3 \\
Sinais de Refluxo Gastresofágico & 23 & 0 \\
\hline
\end{tabular}

Tabela 7. Achados nos pacientes portadores de vasculodisgenesia.

\begin{tabular}{lcc}
\hline Idade & $\begin{array}{c}24 \text { a } 72 \\
\text { anos }\end{array}$ & $\begin{array}{c}\text { Média }=41,68 \\
\text { anos }\end{array}$ \\
\hline Sexo & $\mathrm{N}$ & $\%$ \\
\hline Masc & 4 & 13 \\
Fem & 28 & 87 \\
\hline \multicolumn{1}{c}{ Lesão Isolada } & $\mathrm{N}$ & $\%$ \\
Lesão Associada & 24 & 75 \\
$\quad$ Cisto & 8 & 25 \\
$\quad$ Sulco & 7 & 88 \\
\hline Fenda Glótica & 1 & 12 \\
\hline$\quad$ Triangular Posterior & $\mathrm{N}$ & $\%$ \\
$\quad$ Triang.médio-posterior & 4 & 13 \\
$\quad$ Dupla & 1 & 3 \\
$\quad$ Ampulheta & 1 & 3 \\
$\quad$ Fusiforme & 0 & 0 \\
$\quad$ Irregular & 3 & 9 \\
$\quad$ Paralela & 1 & 3 \\
Sinais de Refluxo Gastresofágico & 3 & 9 \\
\hline
\end{tabular}

A presença de ponte mucosa foi identificada em apenas 1 dos 1093 pacientes avaliados, sendo o paciente do sexo masculino. A lesão estava associada a sinais de cordite bilateral, sulco tipo bolsa em prega vocal esquerda e cisto em prega vocal direita, associada à fenda glótica do tipo irregular e sem sinais sugestivos de refluxo gastresofágico.
Tabela 6. Achados nos pacientes portadores de pólipo de pregas vocais.

\begin{tabular}{lcc}
\hline Idade & $\begin{array}{c}20 \text { a } 77 \\
\text { anos }\end{array}$ & $\begin{array}{c}\text { Média }=45,57 \\
\text { anos }\end{array}$ \\
\hline Sexo & $\mathrm{N}$ & $\%$ \\
\hline Masc & 13 & 17 \\
Fem & 62 & 83 \\
\hline & $\mathrm{N}$ & $\%$ \\
\hline Lesão Isolada & 69 & 92 \\
Lesão Associada & 6 & 8 \\
$\quad$ Edema de Reinke & 3 & 50 \\
Vasculodisgenesia & 2 & 33 \\
$\quad$ Sulco & 1 & 17 \\
\hline Fenda Glótica & $\mathrm{N}$ & $\%$ \\
\hline \multicolumn{1}{c}{ Triangular Posterior } & 0 & 0 \\
Triang.médio-posterior & 0 & 0 \\
Dupla & 4 & 5 \\
Ampulheta & 5 & 7 \\
Fusiforme & 5 & 7 \\
Irregular & 28 & 37 \\
Paralela & 3 & 4 \\
Sinais de Refluxo Gastresofágico & 20 & 27 \\
\hline
\end{tabular}

Tabela 8. Achados nos pacientes portadores de paralisia de prega vocal.

\begin{tabular}{lcc}
\hline Idade & $\begin{array}{c}1 \text { a } 78 \\
\text { anos }\end{array}$ & $\begin{array}{c}\text { Média }=55,63 \\
\text { anos }\end{array}$ \\
\hline Sexo & $\mathrm{N}$ & $\%$ \\
\hline Masc & 11 & 33 \\
Fem & 22 & 67 \\
\hline & $\mathrm{N}$ & $\%$ \\
\hline Prega Vocal Direita & 7 & 21 \\
Prega Vocal Esquerda & 16 & 49 \\
Paralisa de Ambas as Pregas & 10 & 30 \\
\hline Fenda Glótica & $\mathrm{N}$ & $\%$ \\
\hline \multicolumn{1}{c}{ Triangular Posterior } & 1 & 3 \\
$\quad$ Triang.médio-posterior & 5 & 15 \\
Dupla & 0 & 0 \\
Ampulheta & 0 & 0 \\
Fusiforme & 0 & 0 \\
$\quad$ Irregular & 8 & 24 \\
$\quad$ Paralela & 1 & 3 \\
\hline
\end{tabular}

\section{DISCUSSÃO}

A incidência de cada uma das lesões benignas das pregas vocais tem sido pouco descrita na literatura. A maioria dos trabalhos são de séries de achados cirúrgicos, o que não representa a real incidência clínica, uma vez que o nódulo, por exemplo, tem indicação cirúrgica restrita ${ }^{3,5,12}$. 
Tabela 9. Achados nos pacientes portadores de leucoplasia de pregas vocais.

\begin{tabular}{lcc}
\hline Idade & $\begin{array}{c}38 \text { a } 78 \\
\text { anos }\end{array}$ & $\begin{array}{c}\text { Média }=54,22 \\
\text { anos }\end{array}$ \\
\hline Sexo & $\mathrm{N}$ & $\%$ \\
\hline Masc & 9 & 53 \\
Fem & 8 & 47 \\
\hline Lesão Isolada & $\mathrm{N}$ & $\%$ \\
Lesão Associada & 11 & 65 \\
$\quad$ Edema de Reinke & 6 & 35 \\
Cisto & 5 & 83 \\
\hline Fenda Glótica & 1 & 17 \\
\hline$\quad$ Triangular Posterior & $\mathrm{N}$ & $\%$ \\
$\quad$ Triang.médio-posterior & 0 & 0 \\
$\quad$ Dupla & 0 & 0 \\
Ampulheta & 0 & 0 \\
Fusiforme & 0 & 0 \\
$\quad$ Irregular & 1 & 6 \\
$\quad$ Paralela & 5 & 29 \\
Sinais de Refluxo Gastresofágico & 2 & 12 \\
\hline
\end{tabular}

Tabela 11. Achados nos pacientes portadores de micromembrana anterior laríngea.

\begin{tabular}{lcc}
\hline Idade & $\begin{array}{c}14 \text { a } 56 \\
\text { anos }\end{array}$ & $\begin{array}{c}\text { Média }=35 \\
\text { anos }\end{array}$ \\
\hline Sexo & $\mathrm{N}$ & $\%$ \\
\hline Masc & 2 & 20 \\
Fem & 8 & 80 \\
\hline Lesão Isolada & $\mathrm{N}$ & $\%$ \\
\hline Lesão Associada & 2 & 20 \\
Cisto & 8 & 80 \\
$\quad$ Nódulo & 5 & 63 \\
$\quad$ Vasculodisgenesia & 2 & 25 \\
\hline Fenda Glótica & 1 & 13 \\
\hline$\quad$ Triangular Posterior & $\mathrm{N}$ & $\%$ \\
$\quad$ Triang.médio-posterior & 0 & 0 \\
Dupla & 0 & 0 \\
$\quad$ Ampulheta & 0 & 0 \\
$\quad$ Fusiforme & 2 & 20 \\
Irregular & 0 & 0 \\
Paralela & 0 & 0 \\
Sinais de Refluxo Gastresofágico & 0 & 0 \\
\hline
\end{tabular}

Bouchayer e colaboradores (1988) apresentam os seguintes achados em uma série de 1283 lesões de pacientes submetidos à microcirurgia laríngea: nódulos, 24\%; cisto, 17\% (14\% epidérmico, $3 \%$ de retenção); sulco vocal, 12\%; pólipo, 11\%; pseudocisto, 6\%; edema de Reinke, 6\%; lesão nodular, 5\%; laringite crônica, 4\%; cicatriz pós-operatória, 3\%; micromembrana anterior, 3\%; granuloma, 1\%; papilomatose laríngea, $<1 \%$; outras lesões, $7 \%$.
Tabela 10. Achados nos pacientes portadores de granuloma de pregas vocais.

\begin{tabular}{lcc}
\hline Idade & $\begin{array}{c}17 \text { a } 61 \\
\text { anos }\end{array}$ & $\begin{array}{c}\text { Média }=40,25 \\
\text { anos }\end{array}$ \\
\hline Sexo & $\mathrm{N}$ & $\%$ \\
\hline Masc & 4 & 22 \\
Fem & 14 & 78 \\
\hline & $\mathrm{N}$ & $\%$ \\
\hline Lesão Isolada & 16 & 89 \\
Lesão Associada & 2 & 11 \\
$\quad$ Vasculodisgenesia & 1 & 50 \\
$\quad$ Paralisia Prega Vocal & 1 & 50 \\
\hline Fenda Glótica & $\mathrm{N}$ & $\%$ \\
\hline$\quad$ Triangular Posterior & 1 & 6 \\
Triang.médio-posterior & 0 & 0 \\
$\quad$ Dupla & 0 & 0 \\
Ampulheta & 1 & 6 \\
Fusiforme & 0 & 0 \\
Irregular & 5 & 28 \\
Paralela & 0 & 39 \\
Sinais de Refluxo Gastresofágico & 7 &
\end{tabular}

Mossallam e colaboradores (1986), em uma série de 106 lesões avaliadas durante a cirurgia, encontraram a seguinte incidência: pólipos, 42\%; cisto, 18\%; edema de Reinke, 14\%; nódulos, 9\%; granuloma, 7\%; neoplasia benignas, $6 \%$; lesões pré-cancerígenas, $4 \%$.

Herrington-Hall e colaboradores (1988) realizaram um estudo retrospectivo com 1262 pacientes, avaliados num período de dois anos, objetivando avaliar a incidência de patologias laríngeas, associadas ao sexo, idade e ocupação. Foram identificadas 22 patologias laríngeas. As mais freqüentes foram: nódulos, 21\%; edema, 14\%; pólipo, 11,4\%; carcinoma, 9,7\%; paralisia de pregas vocais, $8,1 \%$; laringite, 4,2\%; leucoplasia, 4,1\%; granuloma, $1 \%$; cisto, $1 \%$, exame normal, $7,9 \%$; outros, $20 \%$.

Desta forma, a literatura considera as lesões organicofuncionais, em especial o nódulo vocal, como a lesão laríngea mais comumente encontrada em pacientes com queixas vocais, segundo a maioria dos autores.

O nódulo é a principal causa de disfonia entre crianças e adolescentes; sendo, a partir dos 15 anos, praticamente exclusiva do sexo feminino. Herrington-Hall e colaboradores (1988) relatam a maior incidência de nódulos em mulheres na faixa etária dos 25 aos 44 anos.

Em nosso estudo, os nódulos não representaram a lesão mais comum e foram encontrados em 90 dos 1093 pacientes (8\%); com idade variando de 4 a 66 anos, com média de 34,4 anos. Observamos uma maior incidência no sexo feminino (87\%). Quanto à coaptação glótica, 74 pacientes (82\%) apresentavam coaptação incompleta com fenda glótica, sendo mais comum a do tipo dupla (37\%), seguida pela triangular médio-posterior (28\%). 
A gênese do nódulo vocal é multifatorial, envolvendo fatores anatômicos predisponentes, características da personalidade e comportamento vocal inadequado. Dentre os fatores anatômicos, destacamos a presença da fenda glótica triangular médio-posterior, em cujo vértice se concentra a energia vibratória durante a fonação, responsável pelo espessamento tecidual. As fendas duplas, na verdade, são fendas triangulares médio-posterior com lesão de mucosa, predominantemente edema localizado, uni ou bilateral, que produz o aparecimento de uma abertura anterior. Provavelmente como estas fendas comumente resultam da associação do padrão feminino de proporção glótica com a síndrome de tensão muscular, os nódulos apresentam maior incidência em mulheres e crianças ${ }^{2}$.

A lesão mais comum em nosso estudo foi o cisto vocal, encontrado em 267 pacientes (24\%); com idade variando de 10 a 80 anos, com uma média de 40 anos. O sexo feminino predominou, $97 \%$ dos casos. Em $84 \%$ dos pacientes a lesão apresentava-se isolada e em 16\% associada, sendo a vasculodisgenesia a lesão mais freqüentemente associada nestes casos (80\%). Observamos fenda glótica em 58,8\% dos casos, sendo a do tipo ampulheta a mais comum, presente em $29 \%$ dos pacientes.

O cisto corresponde a uma alteração estrutural mínima da cobertura das pregas vocais, sendo um desarranjo estrutural ocorrido na embriogênese e que pode se manifestar em qualquer idade, geralmente disparado pelo uso intenso ou abusivo da voz.

O cisto nos estudos de Mossallam e colaboradores (1986) e Bouchayer e colaboradores (1988) aparece em segundo lugar, em termos de freqüência. Herrington-Hall e colaboradores (1988) diagnosticaram cisto vocal em apenas 12 de 1262 pacientes (1\%) disfônicos, predominando na faixa etária de 45 a 64 anos, sendo 7 homens para 5 mulheres. Provavelmente, questões metodológicas de avaliação laríngea podem explicar parte das diferenças nos resultados.

O edema de Reinke foi a segunda lesão mais freqüente em nosso estudo, ocorrendo em 113 pacientes (10\%). Mossallam e colaboradores (1986) encontraram uma incidência de $14 \%$, enquanto Bouchayer e colaboradores (1988), de 6\%. A idade dos pacientes variou de 26 a 76 anos, com média de 51 anos, sendo mais comum em mulheres (96\%), o que corresponde aos achados da literatura, que apontam o sexo feminino como prevalente nesta patologia. Apenas 17\% dos pacientes apresentavam lesão associada, sendo a mais comum a leucoplasia, 35\% dos casos. Encontramos coaptação incompleta em 32\% dos pacientes, sendo a fenda irregular a mais comum (25\%), devido às características da própria massa desta lesão. Herrington-Hall e colaboradores (1988) encontraram edema em 272 dos 1262 pacientes avaliados (14,1\%), com predomínio também no sexo feminino na faixa etária de 25 a 44 anos, seguida pela faixa de 45 a 64 anos.
Já o sulco vocal foi encontrado em 110 pacientes (10\%). Bouchayer e colaboradores (1988), analisando 1283 pacientes, encontraram uma incidência de sulco vocal de $12 \%$, muito próxima ao nosso resultado. A identificação desta lesão, assim como do cisto vocal, depende, em grande parte, do conhecimento do avaliador sobre sua existência e principais características ${ }^{2,7}$.

Os pólipos foram encontrados em $7 \%$ dos pacientes, com idade variando de 20 a 77 anos, com média de 45 anos, sendo mais comum em mulheres (83\%), o que não corresponde aos achados de outros autores, que chegam até mesmo a considerar esta lesão como característica do sexo masculino. Kleinsasser (1982), em revisão de 900 casos de pólipos de pregas vocais, encontrou uma predominância no sexo masculino (76\%), com idade média de 40 anos em homens e 38 anos nas mulheres.

Observamos vasculodisgenesia em $4 \%$ dos pacientes, sendo que em $76 \%$ dos casos aparecia como lesão isolada, o que não corresponde aos dados da literatura, onde raramente esta lesão é identificada isoladamente ${ }^{3,6,12}$.

As paralisias de pregas vocais foram responsáveis por 3\% dos casos de disfonia, com uma maior incidência em pacientes com média de idade de 55 anos, conforme já citados por outros autores. Herrington-Hall e colaboradores (1988) encontraram uma incidência de 8,1\% de paralisias, predominando na faixa etária mais tardia, acima dos 64 anos.

A leucoplasia foi identificada em 17 pacientes (2\%), com uma idade média de 54 anos, sem diferença significativa quanto ao sexo ( 9 homens para 8 mulheres), o que também discorda da maioria dos autores, que cita uma maior incidência em indivíduos do sexo masculino. Herrington-Hall e colaboradores (1988) encontraram uma incidência de leucoplasia de 4,1\%, com predomínio do sexo masculino e da faixa etária de 45 a 64 anos.

O granuloma foi encontrado em 18 pacientes (2\%), com idade média de 40 anos, com predomínio do sexo feminino (78\%), o que difere dos dados de literatura, onde esta lesão é mais comumente encontrada em pacientes do sexo masculino. Bouchayer e colaboradores (1988) e Herrington-Hall e colaboradores (1988) encontraram uma incidência de $1 \%$, enquanto Mossallam e colaboradores (1986) citam uma incidência de 7\%. Lehmann e Widman (1986) analisaram uma série de 1300 pacientes com disfonia, encontrando uma incidência de 5\% de granuloma, com uma idade média de 44 anos.

Por sua vez, a micromembrana anterior foi identificada em 10 pacientes $(<1 \%)$. Bouchayer e colaboradores (1988) encontraram uma incidência de 3\%. Em $80 \%$ dos casos do nosso estudo, a lesão estava associada a uma outra patologia, sendo o cisto vocal a associação mais comum. Estes dados não correspondem à literatura, que relata mais freqüentemente a ocorrência 
conjunta de micromembrana anterior e leucoplasia ou nódulos vocais.

Quanto à presença de sinais sugestivos de refluxo gastresofágico, o grupo que apresentou uma maior incidência foi o dos pacientes com diagnóstico de leucoplasia - 53\%; seguido por vasculodisgenesia, 50\%; granuloma, 39\%; edema, 35\%; sulco, 31\%; pólipo, 27\%; nódulo, 26\% e cisto, 23\%. Classicamente, o refluxo gastresofágico está mais comumente relacionado à laringite e ao granuloma laríngeo posteriores. Ohmann e colaboradores (1983) encontraram uma incidência de refluxo gastresofágico em $74 \%$ dos pacientes com granuloma de pregas vocais contra 30\% da população geral. Entretanto, Lehmann \& Widman (1986) identificaram refluxo gastresofágico em apenas 1 de 32 pacientes com granuloma de contato. O refluxo gastresofágico ainda está associado à estenose laríngea e traqueal, laringosespasmos, asma, síndrome da morte súbita da infância, laringomalacea, displasia broncopulmonar e pneumonias aspirativas.

\section{CONCLUSÕES}

Através do estudo dos resultados deste trabalho, que analisou exames videolaringoscópicos de 1093 pacientes atendidos de março de 1999 a março de 2000, podemos concluir que a lesão laríngea mais freqüente em pacientes com queixas vocais foi o cisto, que se configura como uma alteração estrutural mínima da cobertura das pregas vocais, levando a uma disfonia funcional, contrariando a maioria dos autores ${ }^{3,8,12}$, que apontam as lesões organofuncionais (nódulo, pólipo e edema de Reinke) como as lesões mais comuns.

\section{REFERÊNCIAS BIBLIOGRÁFICAS}

1. BASTIAN, R.W. - Benign Vocal Fold Mucosal Disorders. In CUMMINGS, C.W. et al. - Otolaryngology Head \& Neck Surefluxo gastresofágicory, Mosby, 1998.

2. BEHLAU, M. S. \& PONTES, P. A. - Avaliação e Tratamento das Disfonias. São Paulo, ed. Lovise, 1995.

3. BOUCHAYER, M.; CORNUT, G. - Microsuegery for benign lesions of the vocal folds. Ear Nose Throat J 67:446-66, 1988

4. BRODNITZ, F.S. - Results and limitantions of vocal rehabilitation. Arch Otolaryngol Head Neck Surg., 77:148, 1963.

5. FAWCUS, M. - Voice Disorders and their management. 2 ed. San Diego, Singular, 1992.

6. GOULD, W.J.; RUBIN, J.S.; YANAGISAWA, E. - Benign Vocal Fold Patologhy Though the Eyes of the Laryngologist. In: SATALOFF, R. T. et al. Diagnoses and treatment of voice disorders. Igaku-Shoin Medical Publishers Inc, New York, 1995.

7. GREISEN, O. - Vocal fold sulcus. J Laryngol. Otol, 98:293-6, 1984.

8. HERRINGTON-HAL, B. L.; STEMPLE, J. C.; NIEMI, K. R. \& MCHONE, M. M. - Description of laryngeal pathologies by age, sex and occupation in a treatment-seeking sample. Journal of Speech and Hearing Disorders, 53:57-64, 1988.

9. KLEINSASSER, O. Microlaryngoscopy and endolaryngeal microsurefluxo gastresofágicory: tecnique and typical findings, ed 2, Baltimore, University Park Press, 1979.

10. KLEINSASSER, O. - Pathogenesis of vocal cord polips. Ann Otol Rhinol Laryngol., 91:378-81, 1982.

11. LEHMAN, W.; WIDMAN, J. J. - Nonspecific granulomas of the larynx. In KIRCHNER, J.A. (ed.) Vocal Fold Histopatbology: A Symposium San Diego, College-Hill, pp. 97-107, 1986.

12. MOSSALLAM, I.; KOTBY, M. N.; GHALY, A. F. et al. - Histopathological aspects of benign vocal fold lesions associated with dysphonia. In KIRCHNER, J.A. (ed.) Vocal Fold histipathology: A symposium. San Diego, College-Hill, pp. 65-80, 1986.

13. OHMAN, L.; OlOFFSON, J.; TIBBLING, L. et al. -Esophageal dysfunction in patients with contact ulcer of the larynx. Ann Otl Rhinol Laryngol., 92:228-30, 1983.

14. RAMIG, L. \& VERDOLINI, K. Treatment efficacy: voice disorders. $J$ Speech Lang. Hear. Res., 41: S101-16, 1998. 